\title{
High Speed Mobility Management Performance in a Real LTE Scenario
}

\author{
Ahmed Abdelsalam M. Kheir Abuelgasim \\ School of Electrical Engineering \\ Universiti Teknologi Malaysia \\ Johor, Malaysia \\ ahmedkhair22@gmail.com
}

\author{
Kamaludin Mohamad Yusof \\ School of Electrical Engineering \\ Universiti Teknologi Malaysia \\ Johor, Malaysia \\ kamalmy@utm.my
}

\begin{abstract}
This paper discusses the mobility management for high-speed users, which is a crucial challenge for all mobile operators, especially when users are moving vertically across different network technologies. Mobility, also known as seamless connectivity, is directly influencing the quality of service (QoS). Mobility management-Handover (HO) performance was evaluated by field measurements of a Long Term Evolution (LTE) network of a mobile operator in the Middle East. Drive test measurements were used to analyze the results of different high-speed scenarios. User Equipment (UE) with high speed of $80-140 \mathrm{~km} / \mathrm{h}$ causes a high risk of failure of seamless connectivity as the $\mathrm{HO}$ procedure timing is longer than for UE with smaller speed. HO failure and call drop may occur when UE is moving with high speed across two adjacent cells in highways. During measurements, HO failure occurred when UE speed was $140 \mathrm{~km} / \mathrm{h}$, and HO preparation timing increased when UE speed increased.
\end{abstract}

Keywords-mobility management; handover; heterogeneous network; user equipment (UE); speed; field measurement

\section{INTRODUCTION}

The most important objective that differentiates cellular technologies is data demand. The design of applications for users mobile users, is a demanding task for mobile telecom operators while continuous communication is constantly required. Mobile operators need to improve user-friendly connectivity, while the challenge of mobility will boom in the future. In a mobile telecom network, user equipment (UE) locations continue to change depending on user behavior, and this UE mobility should be supervised through the mobile network so that incoming calls are delivered to the UEs. Mobility creates handover (HO) as a result of UE moving from one place to another. The HO should be completed regardless of the network access technology. Operators may also need to seamlessly combine several technologies that deliver unlimited content to customers in a global environment where fourthgeneration $(4 \mathrm{G})$ or long-term evolution (LTE) networks and beyond are beginning to spread. To deliver "seamless" interoperability and convergence between these heterogeneous systems, the integration of unique wireless network technology is needed, and therefore the use of vertical handover (VHO) methods is required [1]. HO, also known as Handoff, occurs when a mobile station (MS) travels through one or more cellular cells and connects to a new cell while disconnecting from the source cellular cell. $\mathrm{HO}$ can be classified according to unique classifications. If the $\mathrm{HO}$ is within identical wireless access technologies, it is called horizontal handover, while the handover known as vertical handover occurs among separate wireless access techniques. HO decisions can be made using several techniques such as fuzzy logic and machine learning to overcome the mobility challenges and reduce the rate of $\mathrm{HO}$ failures and delay. Moreover, to optimize the cellular network, hybrid algorithms of $\mathrm{HO}$ decisions can use tree methodology to learn the coverage of cells, and predict if the HO is necessary to be done $[2,3]$. The LTE platform allows mobility, according to the 3rd Generation Partnership Project (3GPP), for low user speeds from 0 to $15 \mathrm{~km} / \mathrm{h}$ and faster user speeds from 15 to $120 \mathrm{~km} / \mathrm{h}$. Mobility at speeds ranging from $120 \mathrm{~km} / \mathrm{h}$ to $350 \mathrm{~km} / \mathrm{h}$ (or up to $500 \mathrm{~km} / \mathrm{h}$ based on the range of frequencies), will effect $\mathrm{HO}$ and performance (i.e. interruption time) will be lower than or equal to that given in the circuit switch (CS) domain handover [4]. The LTE must minimize delay and packet loss in voice transmission to fulfill this intention and assure reliability in high-speed data transmission circumstances.

Heterogeneous wireless networks can incorporate various radio access technologies such as Global System for Mobile (GSM), General Packet Radio Service (GPRS), High Speed Packet Access (HSPA), Universal Mobile Telecommunications System (UMTS), or LTE. The vital promise of interconnecting these heterogeneous networks is to improve efficiency by achieving high data rates and supporting high-quality video, streaming, and multi-casting [5]. It is also identified as a network made of a mixture of high-power and low-power stations, some of which are configured with limited access, while others may lack wired backhaul. Heterogeneous networks are networks that use a combination of classical macrocells, pico, femto, or relay nodes. In addition to possibly having a hybrid of closed and open user access, this type of networks is described by clear variations in the transmission power used by different network nodes. In practice, such power differences position the low-power nodes (pico, femto, and relay) at a disadvantage over the high-power nodes (macrocells). A heterogeneous network requests higher frequencies relying on the relationship between cell radius size and frequency. mmWaves have been recently introduced in 
International Mobile Telecommunications (IMT) and are identified as above $6 \mathrm{GHz}$, due to the strong demand for extremely high bandwidth (up to $1 \mathrm{GHz}$ ) and high data volumes, mainly in crowded urban areas. High frequencies above $6 \mathrm{GHz}$ are essential to be used in IMT [6].

\section{LITERATURE REVIEW}

Most of the researches addressed here are using received signal strength and UE speed as main criteria in the HO procedure. Along with signal strength, some researchers combine additional parameters to get more mobility efficiency, such as signal quality, UE speed, available bandwidth, and spectrum of cells to reduce HO failure, call drop, HO delay, and improve overall HO performance and QoS. In [7], authors showed the importance of signal strength using a HO scheme to assess system results in terms of several HOs, the period between two subsequent HOs, and SINR uplink. The HO based on the analysis of signal strength was explored at varying UE speeds from 3 to $120 \mathrm{~km} / \mathrm{h}$. The primary goal of the study was to reduce the overall number of HOs by increasing time-totrigger (TTT) and reducing an average of SINR. Furthermore, based on the results of the measurement HO bandwidth, the margin for distinct UE speeds has been evaluated. The study was concentrated on the influence of signal strength using a system performance evaluation algorithm. Authors in [8] assessed HO effectiveness when implementing different TTT approaches in the LTE scheme. The proposed study intended to decrease ping-pong HO, yet due to delay in the HO, TTT may trigger radio link failure (RLF). Two procedures were used to achieve optimal HO: The adaptive technique reached by flexibility choice of the TTT value for each UE velocity depending on an RLF rate of $2 \%$ and the grouping technique accomplished by categorizing UE velocities into three groups and applying the right TTT value to each range. If the SINR in the serving cell becomes stronger than in the target cell, then the HO will never be practiced, and this occurrence will be marked as "leaving the event". UE expected velocity sets of 3 , $15,30,60$ and $120 \mathrm{~km} / \mathrm{h}$ over macro-macro HO and macro-pico HO. The RLF rate increases as a consequence and ping-pong declines as UE velocity increases. The RLF rate remains stable when the TTT value exceeds $5120 \mathrm{~ms}$, but when the UE speed is $120 \mathrm{~km} / \mathrm{h}$, the RLF increases. On the other hand, at $120 \mathrm{~km} / \mathrm{h}$ UE speed, the ping-pong reduces. Authors in [9] also explored the impact of high-speed mobility on three handover algorithms' output in LTE. They used the A3 RSRP event with varying UE speed ranges $(0-3 \mathrm{~km} / \mathrm{h}, 4-60 \mathrm{~km} / \mathrm{h}, 60-120 \mathrm{~km} / \mathrm{h}$ and over $120 \mathrm{~km} / \mathrm{h}$ ). Power Budget Handover is an efficient algorithm that utilizes two parameters to create $\mathrm{HO}$ decisions since it uses TTT and the handover margin (HOM). HOM is a variable representing the boundary for the signal strength difference between the serving cell and the target cell. The signal strength of the target cell must be larger than the signal strength of the serving cell plus the HO margin, therefore, the eNodeB can consider the handover of UE. By stopping the handover trigger within a certain moment, the TTT is used to prevent ping-pong so that the $\mathrm{HO}$ procedure is done only after TTT is fulfilled. The UE measures the signal strength that includes antenna gain, route loss, and fast fading average along with bandwidth measurement in Received Signal Strength Based TTT. The signal strength is measured periodically, while the variations between the Reference Signal Received Power (RSRP) serving and the target cell are incorporated in the Integrator Handover algorithm using Infinite Impulse Response (IIR) filter, and the $\mathrm{HO}$ is made according to the triggering situation between the variations between the RSRP values. To assess the impact of mobility in the above three schemes, UE roams across the network at $3,30,60,120$ and $170 \mathrm{~km} / \mathrm{h}$ randomly chosen speeds. The efficiency was evaluated at every speed. The increase in speed in the range of $60-120 \mathrm{~km} / \mathrm{h}$ had a mildly stronger performance, and when the speed exceeded $120 \mathrm{~km} / \mathrm{h}$, the algorithm worked best with a $120 \mathrm{~dB}$ SNR.

In [10], field measurement was used in two separate zones (urban and highway) to analyze mobility efficiency and timing of HO in both slow and high velocity environments. The researchers estimated both $\mathrm{HO}$ preparation time and $\mathrm{HO}$ execution time, most likely equivalent to the interruption time of HO. Regarding HO timing, other key performance indicators (KPIs) such as intra number, inter-site handover, amount of RLFs, and HO failure rate were mentioned. During the drive tests, the proposed algorithm utilized RSRP and UE velocity as the primary parameter. Before the HO command measurement $1800 \mathrm{MHz} H O$ was initiated using the A3 event where RSRP, TTT, and offsets were used. Three values of TTT were used $(320,1024$, and $1280 \mathrm{~ms})$. Larger TTT values tend to postpone HOs, while shorth TTT values result in quicker and more aggressive HOs. The amount of $\mathrm{HO}$ rises during high speed, and the use of Mobility Robustness Optimization (MRO) decreases $\mathrm{HO}$ failures. The average duration of the $\mathrm{HO}$ process was $77 \mathrm{~ms}$ at a standard velocity in the town, whereas it lasts for 65 and $69 \mathrm{~ms}$ at a speed of 80 and $100 \mathrm{~km} / \mathrm{h}$ respectively on the highway. The average time of $\mathrm{HO}$ interruption was between 24 and $29 \mathrm{~ms}$. However, occasional cases of delays greater than $100 \mathrm{~ms}$ were reported.

\section{High-SPEED MOBILITY CHALLENGES}

At present, a cellular network consists of both new and old technology because when new technology standards are released and are commercially launched, not all of the cellular networks and legacy technology will be replaced at once. In other words, the cellular system works with different technologies, which means differences between network, e.g. the spectrum or cell size of each technology might be different. Also, the mobility between different cellular technologies might be different, therefore, Heterogeneous Networks (HetNets) which may consist of $3 \mathrm{G}$, LTE-4G and future generation $(5 \mathrm{G})$, are introduced to fill the customers' needs of high coverage, reliability, seamless connectivity, and high QoS. Heterogeneous networks are very congested with UEs and personal digital assistants (PDAs). They are very complicated and therefore more challenges must be addressed. Mobility and spectrum limitation are the most challenging topics for the HetNets and upcoming mobile generations. All mobile carriers around the world are fighting to hold enough spectrum to be able to serve their customers and provide services within different technologies. Moreover, many regulatory bodies around the world have already started to allocate spectrum via auctions, resulting in driving costs to very high level, thus mobile operators have to utilize the allocated spectrum properly. Having successful HOs is considered a big challenge 
when the UE moves vertically between different technologies in a HetNet. Mobility in a HetNet is considered a critical component that directly impacts mobile network QoS in terms of $\mathrm{HO}$, call drop percentage, and seamless connectivity. HO delay is one of the major HetNet challenges when cells have different radius sizes. Highway mobility is considered as a most annoying issue for mobile operators because the high speed of the UE (which may reach $120-140 \mathrm{~km} / \mathrm{h}$ which is the maximum speed limit for most countries [11, 12]). In such cases, mobile operators have to consider the UE velocity in $\mathrm{HO}$ preparation. Also, the inter-site distance between two adjacent cells in a highway should be included in the measurements. Therefore, HO delay due to additional required measurements is another challenge for high-speed mobility. Figure 1 illustrates an UE moving with high speed between two adjacent cells.

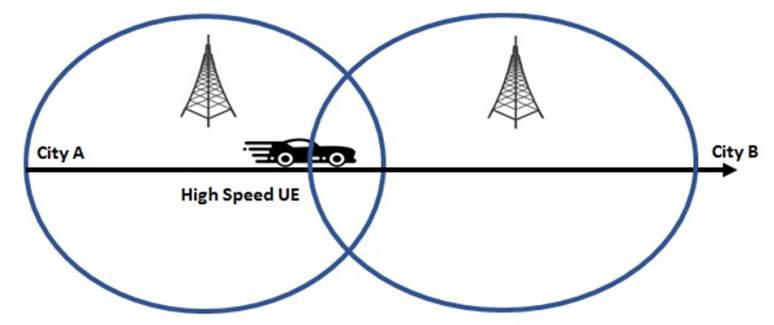

Fig. 1. High-speed UE in an LTE network

\section{HANDOVER PROCEDURE AND TIMING}

The LTE HO procedure can be seen in Figure 1 of [10]. UE is configured to do measurements periodically, and the measurement report period is set by the operator ( $3 \mathrm{~ms}$ in most operators). These measurement reports are sent to the serving cell where UE has been currently serving. Once a measurement report has been sent by the UE to the source cell that decides to handover, the origin cell sends a request for $\mathrm{HO}$ to the destination cell that selects whether or not the UE can be handed over to the cell depending on the control of admission. Then the acknowledgment of the HO request is communicated to the source cell to verify that the new cell can accommodate the UE. Additionally, the Handover command, also known as the reconfigure message for Radio Resource Control (RRC) connection, is sent to UE to indicate that $\mathrm{HO}$ is going to take place. Therefore, the HO preparation time is defined as the elapsed time between the moment of a measurement report and the moment when the UE receives RRC connection reconfigure message. Then, the UE is disconnected from the serving cell and initiates the synchronization procedure with the new cell, the data communication is paused and restored when the complete message is obtained with the handover setup or RRC connection reconfiguration. Therefore, the $\mathrm{HO}$ interruption time is defined as the time interval between both RRC messages. $\mathrm{HO}$ interruption time is also known as $\mathrm{HO}$ execution time or accomplishment time. However, authors in [13] declared that the data interruption time during the $\mathrm{HO}$ is slightly longer than the $\mathrm{HO}$ execution time as the target cell begins scheduling the data for the UE after the HO configuration message is broadcasted. The overall $\mathrm{HO}$ time can be defined as the time interval between the instant when the UE directs the measurement reports and the instant when data is restored for the UE.

\section{MEASUREMENTS}

Handover field measurements have been performed for a telecom operator cellular network using drive test tool and were analyzed by the use of Actix software. Drive test measurements were performed on a highway between two cities. Drive tests were undertaken in various UEs for speeds ranging from $80 \mathrm{~km} / \mathrm{h}$ to $140 \mathrm{~km} / \mathrm{h}$. The Samsung Galaxy S8 terminal was used in the measurement and was categorized as category 16. UEs were LTE capable, supporting speeds up to $800 \mathrm{Mbps}, 256$ QAM modulation, $4 \times 20 \mathrm{MHz}$ carrier aggregation (CA), and were able to work with the $1800 \mathrm{MHz}$ band. LTE cells were configured to operate in both TimeDivision-Duplex (TDD) and Frequency-Division-Duplex (FDD) modes using separated ports to ensure compatibility to both TDD and FDD UE types. Nokia BTS Site Manager was used to get LTE cell setup where eNB carrier frequency was $1870 \mathrm{MHz}$ for downlink and $1775 \mathrm{MHz}$ for uplink, both operating with $20 \mathrm{MHz}$ of bandwidth. The cell was using $4 \times 4$ MIMO, and no virtual antenna mapping technique was used. Measurements were conducted in both directions between A and $\mathrm{B}$ which can be defined as the starting point and ending point respectively. The measurement results were analyzed to extract crucial parameters such as Reference Signal Received Power (RSRP), Reference Signal Received Quality (RSRQ), Cell ID, and RRC connection configuration messages.

\section{EXPERIMENTAL RESULTS}

\section{A. Coverage Area}

The cell coverage measured during the driving test ranges from $0.4 \mathrm{~km}$ and $0.7 \mathrm{~km}$, to $2.8 \mathrm{~km}$ for LTE cells on the same highway at separate places.

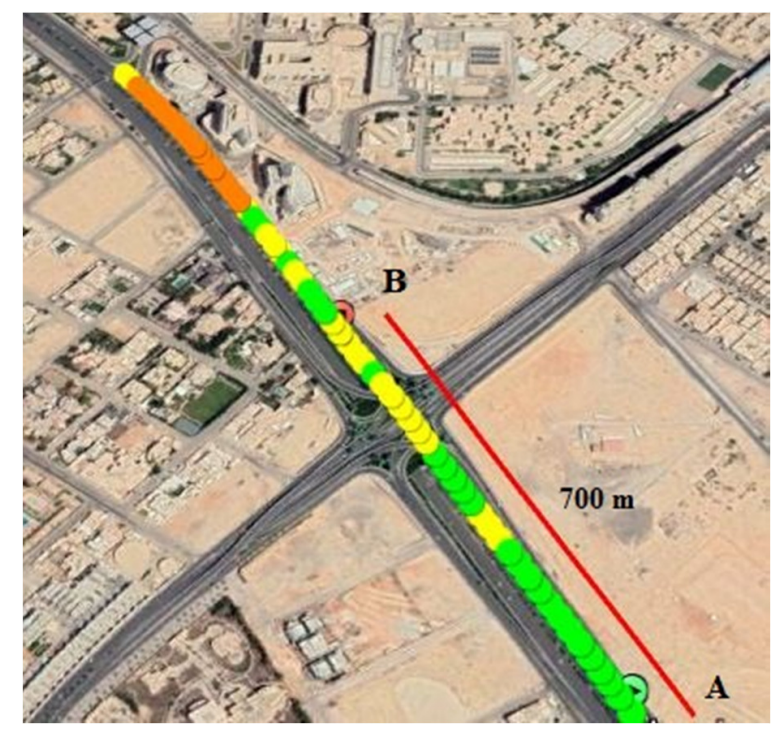

Fig. 2. The observated high-density area of the city (screenshot from Google Earth, (C) 2018 Google, Image (C) 2019 Maxar Technologies, Image (C) 2019 CNES/Airubus) 
Low power cells were used by a mobile operator in the downtown zone where high capacity and seamless service are required in a high-density region, whereas cell power rises towards the highway that runs outside the city where the lowdensity zone is located, so the cell transmission range is also improved. In the city's high-density region, the reported cell coverage was $400 \mathrm{~m}$ and $700 \mathrm{~m}$, while a low-density region it was assessed at $2800 \mathrm{~m}$. Figures 2 and 3 show the observation area during the drive test in the direction from the city toward outside it.

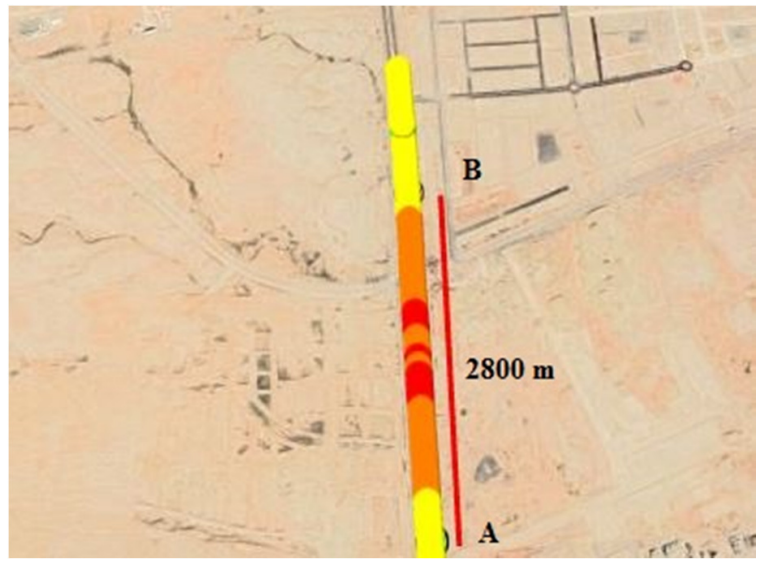

Fig. 3. The observated low-density area (screenshot from Google Earth, (C) 2018 Google, Image (C) 2019 Maxar Technologies, Image (C) 2019 CNES/Airubus)

\section{B. RRC Message Analysis Or Measurement Report Analysis}

Based on RRC message analysis, the UE is configured to send measurement reports periodically. RSRP, RSRQ, and neighboring cells are included in these reports. The measurements of RSRP and RSRQ are used to trigger HOs from the serving to the target cell. Figures 4 and 5 show the RSRP and RSRQ during the measurement report for UE speed of $140 \mathrm{~km} / \mathrm{h}$. The RSRP value where the HO must be conducted is set by the mobile operator to be within $-94 \mathrm{dBm}$ and $-102 \mathrm{dBm}$ based on the field measurement, therefore, optimization robustness is used at the network.

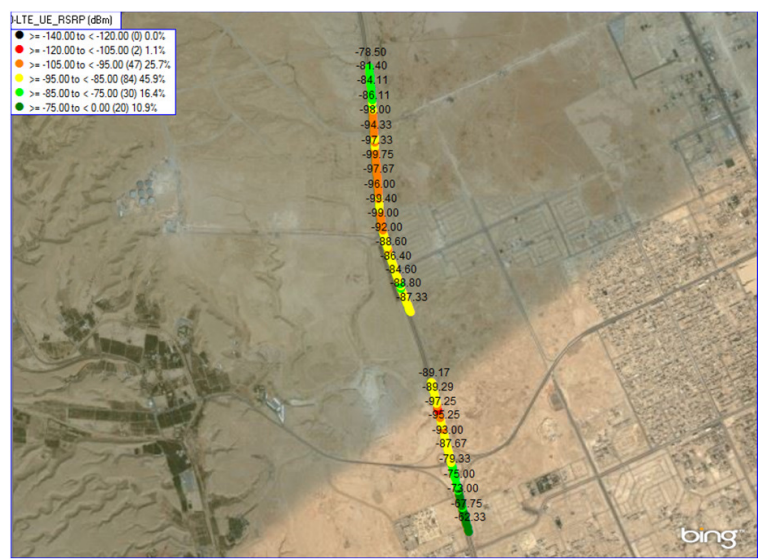

Fig. 4. RSRP measurements when UE speed is $140 \mathrm{~km} / \mathrm{h}$ (screenshot from Google Earth, (C) 2018 Google, Image (C 2019 Maxar Technologies, Image (C) 2019 CNES/Airubus)
The measured RSRP value is set within the range of $-80 \mathrm{dBm}$ and $-105 \mathrm{dBm}$ and the RSRP HO value to a bit higher value in order to keep QoS in a high level and avoid call drops, although call drops occurred during our test when UE speed was $140 \mathrm{~km} / \mathrm{h}$. Figure 6 clearly shows that there is a failure in RSRP value when it varies against HO time, resulting in a call drop. The Figure illustrates the RSRP of the serving cell before and after the $\mathrm{HO}$.

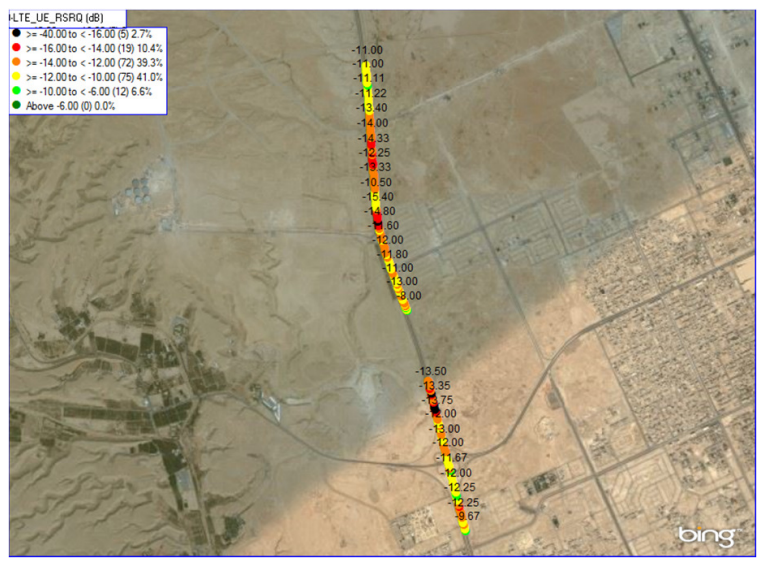

Fig. 5. RSRQ measurements when user equipment (UE) speed is 140 $\mathrm{km} / \mathrm{h}$ (screenshot from Google Earth, (C) 2018 Google, Image (C) 2019 Maxar Technologies, Image (C) 2019 CNES/Airubus)

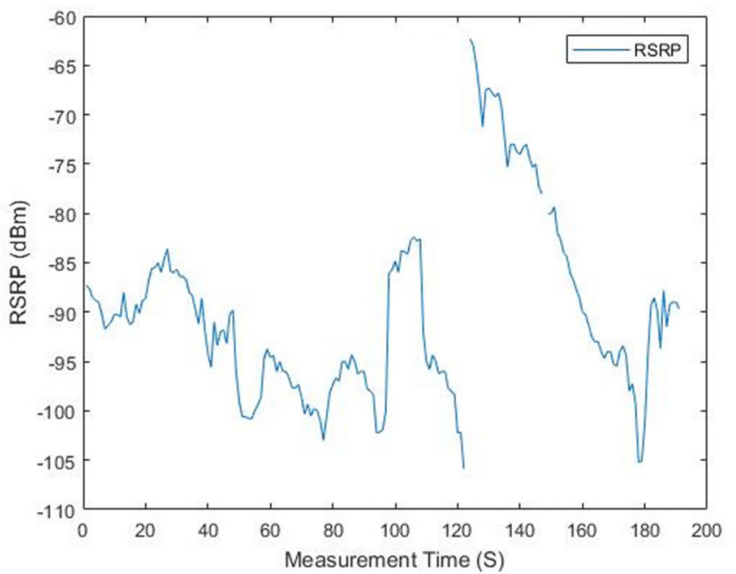

Fig. 6. RSRP variation when user equipment (UE) speed is $140 \mathrm{~km} / \mathrm{h}$

\section{HO Events and Timing During Measurements}

During the driving test, the number of frequent HOs increases when the UE's velocity increases, however, using high power cells helps reducing $\mathrm{HO}$ numbers as cell coverage is wider than small cells in the city center. Frequent HOs are experienced during the drive tests with UE speed between 100 and $140 \mathrm{~km} / \mathrm{h}$. In the driving test, the direction and location of the selected sites play a significant part. An inter-frequency $\mathrm{HO}$ has been recorded when UE rides from the city with $140 \mathrm{~km} / \mathrm{h}$ velocity, whereas the received signal strength has been much better when the UE velocity is higher than $140 \mathrm{~km} / \mathrm{h}$, with the UE driving toward the city and is closer to site location. HO timing was analyzed from the measurement 
results and it was observed that the $\mathrm{HO}$ preparation time increases as UE speed increases. Moreover, the HO execution time is slightly faster than the HO preparation time. Also, the experienced HO time values on the highway are mostly higher than in the city. Figure 7 illustrates the change of the HO timing with UE velocity. The $\mathrm{x}$-axis illustrates user speed $(\mathrm{km} / \mathrm{h})$ while the y-axis illustrates measurement time (s).

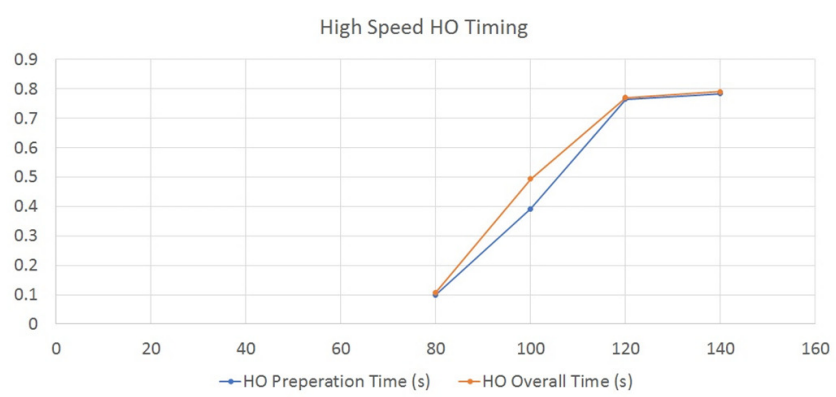

Fig. 7. HO timing vs UE velocity

\section{CONCLUSION}

In this paper, high-speed mobility performance and $\mathrm{HO}$ timing were investigated through drive test measurements for a real situation in a mobile operator's LTE network. High-speed mobility was performed for various UE speeds ranging from $80 \mathrm{~km} / \mathrm{h}$ to $140 \mathrm{~km} / \mathrm{h}$. Field measurements were analyzed when UE speed was $140 \mathrm{~km} / \mathrm{h}$. The results show that the UE speed directly influences mobility management LTE and hence the QoS. Moreover, the analyzed results show that HO preparation time increases when UE speed increases, and HO execution time is slightly faster than HO preparation time. The overall HO time was found to be below 1s for high-speed UE. Traffic, safety, and real-time applications are directly affected by $\mathrm{HO}$ delay which is higher on highways.

\section{REFEFENCES}

[1] J. Marquez-Barja, C. T. Calafate, J. C. Cano, P. Manzoni, “An overview of vertical handover techniques: Algorithms, protocols and tools", Computer Communications, Vol. 34, No. 8, pp. 985-997, 2011

[2] N. C. Eli-Chukwu, J. M. Aloh, C. O. Ezeagwu, "A systematic review of artificial intelligence applications in cellular networks", Engineering, Technology \& Applied Science Research, Vol. 9, No. 4, pp. 4504-4510, 2019

[3] M. K. Hassan, A. Babiker, M. B. M. Amien, M. Hamad, "SLA management for virtual machine live migration using machine learning with modified kernel and statistical approach", Engineering, Technology \& Applied Science Research, Vol. 8, No. 1, pp. 2459-2463, 2018

[4] 3GPP/ETSI, (TR 25.913Mike) Universal Mobile Telecommunications System (UMTS); LTE; Requirements for Evolved UTRA (E-UTRA) and Evolved UTRAN (E-UTRAN), 3GPP TR 25.913 version 9.0.0 Release 9, ETSI, 2010

[5] M. Zekri, B. Jouaber, D. Zeghlache, "A review on mobility management and vertical handover solutions over heterogeneous wireless networks", Computer Communications, Vol. 35, No. 17, pp. 2055-2068, 2012

[6] ITU, The technical feasibility of IMT in the bands above $6 \mathrm{GHz}$, Technical Report Rep. ITU-R M.2376-0, ITU, 2015

[7] M. Anas, F. D. Calabrese, P. E. Mogensen, C. Rosa, K. I. Pedersen, "Performance evaluation of received signal strength based hard handover for UTRAN LTE", IEEE 65th Vehicular Technology Conference, Dublin, Ireland, April 22-25, 2007
[8] Y. Lee, B. Shin, J. Lim, D. Hong, "Effects of time-to-trigger parameter on handover performance in SON-based LTE systems", 2010 16th AsiaPacific Conference on Communications, Auckland, New Zealand, October 31-November 3, 2010

[9] M. O. Osifeko, A. A. Okubanjo, O. R. Abolade, O. K. Oyetola, A. O. Oyedeji, O. I. Sanusi, "Evaluating the effect of mobility speed on the performance of three handover algorithms in long term evolution networks", Journal of Applied Sciences and Environmental Management, Vol. 22, No. 4, pp. 503-506, 2018

[10] L. C. Gimenez, M. C. Cascino, M. Stefan, K. I. Pedersen, A. F. Cattoni, "Mobility performance in slow-and high-speed LTE real scenarios", IEEE 83th Vehicular Technology Conference, Nanjing, China, May 1518,2016

[11] B. Katz, J. Ma, H. Rigdon, K. Sykes, Z. Huang, K. Raboy, Synthesis of variable speed limit signs, Federal Highway Administration, 2017

[12] A. Merwaday, I. Guvenc, "Handover count based velocity estimation and mobility state detection in dense HetNets", IEEE Transactions on Wireless Communications, Vol. 15, No. 7, pp. 4673-4688, 2016

[13] S. Barbera, K. I. Pedersen, C. Rosa, P. H. Michaelsen, F. Frederiksen, S. Shah, A. Baumgartner, "Synchronized RACH-less handover solution for LTE heterogeneous networks", 2015 International Symposium on Wireless Communication Systems, Brussels, Belgium, August 25-28, 2015 